\title{
MATRIX-VALUED POSITIVE DEFINITE KERNELS GIVEN BY EXPANSIONS: STRICT POSITIVE DEFINITENESS
}

\author{
W. Franca And V. A. Menegatto*
}

\begin{abstract}
Matrix functions of the form $(x, y) \in \Omega \times \Omega \mapsto \Sigma_{\alpha} A_{\alpha} f_{\alpha}(x, y)$, in which $\Omega$ is a nonempty set, the $A_{\alpha}$ are positive semi-definite matrices of the same fixed order, the $f_{\alpha}$ are complex-valued positive definite kernels on $\Omega$, and the series is convergent for all $x$ and $y$ in $\Omega$ define matrix-valued positive definite kernels on $\Omega$. Here, the sum may be multi-indexed, $\Omega$ may be endowed with either a topological or a metric structure, and $\left\{f_{\alpha}\right\}$ may inherit properties attached to the setting. In this paper, we present a criterion that establishes an abstract necessary and sufficient condition in order that the kernel is strictly positive definite on $\Omega$. We point some implications and connections of the criterion in some relevant and concrete settings in order to motivate future work on the topic.
\end{abstract}

Mathematics subject classification (2020): 42A82, 42C10, 43A35.

Keywords and phrases: Matrix-valued kernels, positive definiteness, kernel expansions, strict positive definiteness, Cauchy-Schwarz inequality.

\section{REFERENCES}

[1] V. S. Barbosa And V. A. Menegatto, Strictly positive definite kernels on compact two-point homogeneous spaces, Math. Inequal. Appl. 19, 2 (2016), 743-756.

[2] C. Berg, J. P. R. Christensen, AND P. Ressel, Harmonic analysis on semigroups. Theory of positive definite and related functions, Graduate Texts in Mathematics 100, Springer-Verlag, New York, 1984.

[3] R. N. Bonfim And V. A. Menegatto, Strict positive definiteness of multivariate covariance functions on compact two-point homogeneous spaces, J. Multivariate Anal. 152, 0 (2016), 237-248.

[4] J. C. Guella And V. A. Menegatto, Strictly positive definite kernels on the torus, Constr. Approx. 46, 2 (2017), 271-284.

[5] J. C. Guella And V. A. Menegatto, Unitarily invariant strictly positive definite kernels on spheres, Positivity 22, 1 (2018), 91-103.

[6] J. C. Guella, V. A. Menegatto, And E. Porcu, Strictly positive definite multivariate covariance functions on spheres, J. Multivariate Anal. 166, 0 (2018), 150-159.

[7] Hongyu He, On matrix valued square integrable positive definite functions, Monatsh. Math. 177, 0 (2015), 437-449.

[8] R. A. Horn and C. R. Johnson, Matrix analysis, second edition, Cambridge University Press, Cambridge, 2013.

[9] Fangyan Lu and Hongwei Sun, Positive definite dot product kernels in learning theory, Adv. Comput. Math. 22, 2 (2005), 181-198.

[10] Tianshi Lu and Chunsheng Ma, Isotropic covariance matrix functions on compact two-point homogeneous spaces, J. Theor. Probab. 33, 0 (2020), 1630-1656.

[11] V. A. Menegatto, Strictly positive definite kernels on the Hilbert sphere, Appl. Anal. 55, 1-2 (1994), 91-101.

[12] M. Michelo AND J. A. Glaunes, Matrix-valued kernels for shape deformation analysis, Geometry, Imaging and Computing 1, 1 (2014), 57-139.

[13] A. PInKUS, Strictly Hermitian positive definite functions, J. Anal. Math. 94, 0 (2004), 3293-318. 
[14] A. PInKus, Strictly positive definite functions on a real inner product space, Adv. Comput. Math. 20, 4 (2004), 263-271.

[15] E. Porcu And V. Zastavnyi, Characterization theorems for some classes of covariance functions associated to vector valued random fields, J. Multivariate Anal. 102, 9 (2011), 1293-1301.

[16] V. L. SHAPIRO, Fourier series in several variables with applications to partial differential equations, CRC Press, Boca Raton, FL, 2011.

[17] G. Szegö, Orthogonal polynomials, fourth edition, Vol. XXIII, American Mathematical Society, Providence, RI, 1975.

[18] D. WitTwar, G. SAntin, AND B. HAaSdonk, Interpolation with uncoupled separable matrixvalued kernels, Dolomites Res. Notes Approx. 11, 0 (2018), 23-29. 